\title{
Temporomandibular dysfunction
}

Jonathan Lomas, Taylan Gurgenci, Christopher Jackson, Duncan Campbell

\author{
Background \\ Orofacial pain is a common \\ presentation in the primary healthcare \\ setting and temporomandibular \\ dysfunction represents one of the major \\ causes. Its aetiology is multifactorial, \\ caused by both masticatory muscle \\ dysfunction and derangement within \\ the temporomandibular joint.
}

\section{Objective}

The aim of this article is to provide an overview of temporomandibular dysfunction, its management and referral considerations for general practioners.

\section{Discussion}

Temporomandibular joint dysfunction affects a large number of adults. Conservative management involving non-pharmacological and pharmacological therapies is effective in the majority of cases.
TEMPOROMANDIBULAR DYSFUNCTION (TMD) encompasses a group of disorders of the masticatory system, broadly divided into muscular conditions and those affecting the temporomandibular joint (TMJ). TMD is a common condition, signs of which appear in up to $60-70 \%$ of the population. ${ }^{1}$ The peak incidence is seen in adults aged 20-40 years. Women are at least four times as likely to suffer from the disorder. ${ }^{1}$ Despite signs of TMD being common, the reported prevalence of symptomatic disease requiring treatment occurs in only $5 \%$ to $12 \%$ of the population. ${ }^{2}$ Broadly speaking, TMD commonly refers to pain involving the TMJ and surrounding structures as well as dysfunction of the joint itself.

\section{Anatomy}

The TMJ is a ginglymoarthrodial joint and is formed by the insertion of the mandibular condyle into the glenoid fossa of the temporal bone. It is a complex synovial system composed of two joints separated by the articular disc. It is the most frequently used joint in the body ${ }^{3}$ and permits a wide range of movements necessary for mastication, swallowing and communication. The relevant musculature involved in TMD are the primary muscles of mastication, including the masseter, temporalis and medial and lateral pterygoid muscles. Sensory innervation of the TMJ involves branches of the third division of the trigeminal nerve, including the auriculotemporal and masseteric nerves. Importantly, the auriculotemporal nerve also provides some sensory innervation to the temporal region, external ear and tympanic membrane, and is highly sensitive to pain responses. ${ }^{4}$

\section{Aetiology}

The aetiology of TMD is poorly understood but is likely to be multifactorial and includes anatomical, pathophysiological and psychosocial factors. Successful management of the disorder involves identifying and managing these predisposing and contributing factors. ${ }^{1}$ Where possible, it is important to distinguish between myofascial causes of TMD and intra-articular disorders of the joint itself. Myofascial disorders are the result of tension, fatigue or spasm of the masticatory muscles, whereas intraarticular disorder stems from mechanical or inflammatory disruption of the joint itself. Musculoskeletal dysfunction is the most common cause of TMD. ${ }^{5}$ Parafunctional behaviours, such as bruxism, teeth grinding, clenching and abnormal posture, stress and anxiety, may all contribute to masticatory muscle pain and spasm. Cognitive and psychiatric disturbance, such as depression and anxiety, and autoimmune disorders, fibromyalgia and other chronic pain conditions are also frequently associated with $\mathrm{TMD}^{6}$ and may signal that the symptoms could be a component of a more complex regional pain syndrome.

Intra-articular causes of TMD include internal joint derangement, osteoarthritis, capsular inflammation, hypermobility and traumatic injury. Inflammatory conditions, such as rheumatoid arthritis and ankylosing spondylitis, can also lead to internal joint derangement. Articular disc displacement from the normal position is the most common intra-articular cause of TMD. ${ }^{7}$ It is important to note, however, that disk displacement is a common finding in the general population and the majority of those are asymptomatic. There is minimal evidence that occlusion abnormalities contribute to TMD.

\section{Differential diagnosis}

It is important to stress that many orofacial and otological conditions can mimic TMD. Conversely, the assumption that 
TMD is the cause of a patient's symptoms can result in a more sinister pathology being missed. Box 1 outlines some of the differential diagnoses to consider when assessing a patient with orofacial pain.

\section{Clinical assessment}

TMD is a clinical diagnosis and a thorough history of the presentation and physical examination should be conducted, paying particular attention to site, onset, character of pain, radiation, duration and associated symptoms. Typically, patients describe pain within the TMJ or mandible as the predominant symptom. This pain may refer to the scalp or neck, and is often exacerbated by mastication, yawning or talking for extended periods. ${ }^{4}$ Difficulty opening the mouth, clicking, popping or crepitus within the TMJ itself and brief locking of the mandible on jaw opening and closure are also often reported. Orofacial pain not associated with jaw movement may suggest another cause for the patient's symptoms. ${ }^{8}$ Headache, particularly tension type headache, is a common presentation in the primary healthcare setting and it is well established that TMD and primary headache disorder are comorbid diseases; ${ }^{9}$ the presence of one may increase the prevalence of the other. Otological symptoms, including otalgia, tinnitus, aural fullness, vertigo and subjective hearing impairment, are also frequently reported in patients with TMD. ${ }^{10}$ These symptoms are more common in patients with myofascial disturbance than in those with intraarticular disc disorder, possibly because of the shared embryonic origin of masticatory muscles and some middle ear structures. ${ }^{11}$

Physical examination should include thorough palpation of the TMJ and masticatory muscles, noting any abnormal mandibular movement, tenderness and signs of bruxism. Normal jaw opening values are $35-45 \mathrm{~mm}$; a value less than $25 \mathrm{~mm}$ suggests dysfunction with no translation occurring within the joint. Examination of the TMJ can be enhanced by placing one finger in the ear canal and palpating the anterior canal wall while the patient opens and closes the mouth. Crepitus experienced at this location is related to articular surface disruption and may suggest the presence of osteoarthritis. Using a stethoscope to ascultate over the TMJ can be a useful adjunct to confirm the presence of crepitus. A clicking or popping sensation when opening the mouth may suggest articular disc displacement. Malocclusion of the teeth should be noted if present; however, this does not contribute to the manifestation of TMD and orthodontic referral is not recommended for this finding alone. In general terms, tenderness elicited on palpation of the TMJ, joint clicking and crepitus are signs of intra-articular derangement, whereas pain on jaw movement, headache and referred pain are suggestive of a muscular problem. ${ }^{12}$ The examination should also include otoscopy to eliminate otological causes, inspection of the oral cavity and palpation of neck musculature.

\section{Diagnostic imaging}

Although TMD is largely a clinical diagnosis, imaging can be useful, particularly when history and examination findings are unclear. Plain radiography and computed tomography may reveal severe degenerative articular disease and, importantly, rule out fractures and dislocations as a cause of the patient's symptoms. Panoramic radiography, including orthopantomogram, is a simple and useful screening tool that should be used early in the diagnostic workup to exclude common odontogenic causes of facial pain as well as assess for joint pathology. Ultrasonography is not sensitive for the diagnosis of intraarticular osteoarthritis, but may be useful in assessment of disc position in TMJ disorders. ${ }^{3}$ Magnetic resonance imaging (MRI) is currently the gold standard for investigation of TMD. The advantage of MRI is the ability to assess soft tissue structures, articular disc displacement and the presence of joint effusion with a high degree of specificity and sensitivity. ${ }^{13}$ Given the high cost of MRI, it is predominantly used in the setting of severe, treatment-resistant TMD, as well as for pre-operative planning purposes; however, the need for medical imaging is becoming less important as surgical options for treatment diminish.

\section{Management}

For the majority of patients, a conservative approach to TMD management should be adopted. Up to $40 \%$ of symptomatic patients have spontaneous resolution of their symptoms without any treatment, ${ }^{14}$ and $50-90 \%$ of patients have relief with conservative therapy. ${ }^{15}$ For the general practioner, non-pharmacological and pharmacological treatments represent the main options available.

Non-pharmacological treatments include patient education and reassurance, jaw rest, a soft diet, warm compress over the region of pain and passive stretching exercises. ${ }^{8}$ Stretching and jaw exercises

\section{Box 1. Causes of orofacial pain}
Dental conditions
- Caries
- Tooth abscess
- Tooth eruption
Otological conditions
- Acute otitis media
- Otitis externa (acute or malignant)
- Mastoiditis
- Eustachian tube dysfunction
Headache disorder
- Tension type headache
- Migraine
- Cluster headache
Neurogenic conditions
- Trigeminal neuralgia
- Postherpetic neuralgia
- Glossopharyngeal neuralgia
Inflammatory conditions
- Temporal arteritis
- Rheumatoid arthritis
- Systemic lupus erythematosus
- Parotitis
Traumatic
- Mandibular fracture/dislocation
- Temporal bone fracture
Other
- Atypical facial pain
- Sinusitis
- Eagle syndrome (stylohyoid syndrome) 
may improve range of movement but may not necessarily improve pain. Given the high degree of association between TMD and cognitive factors, education and reassurance are particularly important and cognitive behavioural therapy is beneficial for short-term and long-term pain management in patients with TMD. ${ }^{16}$ Behaviour modifications, including improving sleep hygiene, stress reduction and elimination of parafunctional habits such as teeth clenching and grinding, are particularly important. ${ }^{8}$ The use of occlusional and non-occlusional splints is controversial and evidence to support their use is inconclusive. ${ }^{17,18}$ They may benefit a select group of patients who have severe bruxism and nocturnal clenching. Referral to a dentist for precise splint-fitting may be considered for these patients, bearing in mind the cost of occlusional splints. Intramuscular botox injections have been shown to be efficacious in myofascial causes of TMD pain and tension-type headache. ${ }^{19}$ TMJ immobilsation has no benefit and may actually worsen symptoms due to muscle contractures and fatigue. Physiotherapy has been shown to be effective in the management of TMD, ${ }^{20}$ especially with regards to improving joint range of motion, and a referral should be considered in refractory cases.

Unless contraindicated, nonsteroidal inflammatory drugs (NSAIDs) represent the first-line pharmacological agents used for acute and chronic pain associated with TMD. Muscle relaxants, such as benzodiazepines, may be useful in patients with recurrent masticatory muscle spasm and chronic bruxism where relaxation techniques are ineffective. ${ }^{12}$ Tricyclic antidepressants, such as amitriptyline, can be trialled as they are often effective in other chronic and regional pain disorders. Medications shown to have little to no benefit in the management of TMD include tramadol, topical preparations such as diclofenac, selective serotonin reuptake inhibitors, serotoninnoradrenaline reuptake inhibitors and monoamine oxidase inhibitors. ${ }^{21}$ Opioids are not recommended for the management of chronic TMD pain because of the risk of the patient developing drug dependency.
Given the high concurrence of cognitive and psychosocial factors affecting many patients with TMD, referral to a pain specialist should be considered for those resistant to conservative measures, particularly when there is suspicion that the patient's symptoms may be part of a more complex regional pain syndrome. Referral to a maxillofacial surgeon should be considered in patients unresponsive to conservative treatment, patients with severe pain and/or dysfunction of the TMJ, and patients with a history of trauma or fracture of the TMJ complex. ${ }^{8}$ Furthermore, patients with persistent or worsening pain, or those with other red flag symptoms (Box 2) with no identifiable source, should be referred to specialist care for further investigation, as rare neoplastic causes such fibrosarcoma and chondrosarcoma of the TMJ, as well as parotid malignancies, have occasionally been misdiagnosed as TMD. ${ }^{22}$ In cases without these symptoms it is reasonable to trial conservative management for six to eight weeks prior to referral.

\section{Conclusion}

Orofacial pain is a common presentation in general practice settings. Clinical assessment and diagnosis of TMD can be achieved in the primary care setting, and in most instances the disorder can be managed using a conservative approach. Imaging is not essential but should be considered when symptoms are severe or when there is a history of trauma. In the majority of instances a trial of conservative therapy should be offered prior to referral to specialist care.

\section{Authors}

Jonathan Lomas BSc, MBBS, Principle House Officer, Department Otolaryngology and Head and Neck Surgery, Toowoomba Hospital, Qld. Jonathan.lomas@ health.qld.gov.au

Taylan Gurgenci BSc, MBBS, General Practitioner Registrar, Pandanus Medical Practice, Capalaba, Qld Christopher Jackson BSc, MBBS, Resident Medical Officer, Department Otolaryngology and Head and Neck Surgery, Toowoomba Hospital, QId

Duncan Campbell BDS, MBChB, MDS(OMS),

GradDipClinDent, FRACDS, FRACDS(OMS), Visiting Medical Officer, Department of Surgery, Toowoomba Hospital, Qld

Competing interests: None.

Provenance and peer review: Not commissioned, externally peer reviewed.

\section{Box 2. Red flag symptoms}

- Persistent and worsening pain

- Trismus

- Cranial nerve abnormalities

- Neurologic dysfunction

- Concurrent infection

- Systemic illness

- Weight loss

- Asymmetrical neck or facial swelling

- Unilateral hearing loss

- Vestibular dysfunction

- New onset or unilateral tinnitus

\section{References}

1. Sharma S, Gupta DS, Pal US, Jurel SK. Etiological factors of temporomandibular joint disorders. Natl J Maxillofac Surg 2011;2(2):116-19. doi: 10.4103/0975-5950.94463.

2. National Institute of Dental and Craniofacial Research. Facial pain. Bethesda, MD: National Institutes of Health, 2014. Available at www. nidcr.nih.gov/DataStatistics/FindDataByTopic/ FacialPain [Accessed 14 October 2017].

3. Ferreira LA, Grossmann E, Januzzi E, de Paula MV, Carvalho AC. Diagnosis of temporomandibular joint disorders: Indication on imaging exams. Braz J Otorhinolaryngol 2016;82(3):341-52. doi: 10.1016/j.bjorl.2015.06.010

4. Rodriguez-Lopez MJ, Fernandez-Baena M, Aldaya-Valverde C. Management of pain secondary to temporomandibular joint syndrome with peripheral nerve stimulation. Pain Physician 2015;18(2):E229-36.

5. Reiter S, Goldsmith C, Emodi-Perlman A, Friedman-Rubin P, Winocur E. Masticatory muscle disorders diagnostic criteria: The American Academy of Orofacial Pain versus the research diagnostic criteria/temporomandibular disorders (RDC/TMD). J Oral Rehabil 2012;39(12):941-47. doi: 10.1111/j.1365-2842.2012.02337.x.

6. Scrivani SJ, Keith DA, Kaban LB. Temporomandibular disorders. N Engl J Med 2008;359(25):2693-705. doi: 10.1056/ NEJMra0802472.

7. de Leeuw R, Klasser GD, editors. Orofacial pain: Guidelines for assessment, diagnosis, and management. 5th edn. Chicago: Quintessence Publishing, 2013.

8. Gauer RL, Semidey MJ. Diagnosis and treatment of temporomandibular disorders. Am Fam Physician 2015;91(6):378-86.

9. Speciali JG, Dach F. Temporomandibular dysfunction and headache disorder. Headache 2015;55(Supp 1):72-83. doi: 10.1111/head.12515.

10. Ramirez LM, Ballesteros LE, Sandoval GP. Topical review: Temporomandibular disorders in an integral otic symptom model. Int J Audiol 2008;47(4):215-27. doi: 10.1080/14992020701843137.

11. Stepan L, Shaw CL, Oue S. Temporomandibular disorder in otolaryngology: Systematic review. J Laryngol Otol 2017;131(S1):S50-56. doi: 10.1017/ S0022215116009191.

12. Buescher JJ. Temporomandibular joint disorders. Am Fam Physician 2007;76(10):1477-82. 
13. Vogl TJ, Lauer HC, Lehnert $T$, et al. The value of $\mathrm{MRI}$ in patients with temporomandibular joint dysfunction: Correlation of MRI and clinical findings. Eur J Radiol 2016;85(4):714-19. doi: 10.1016/j.ejrad.2016.02.001.

14. Garefis P, Grigoriadou E, Zarifi A, Koidis PT. Effectiveness of conservative treatment for craniomandibular disorders: A 2-year longitudinal study. J Orofac Pain 1994;8(3):309-14.

15. Indresano A, Alpha C. Nonsurgical management of temporomandibular joint disorders. In: Fonseca RJ, Marciani RD, Turvey TA, editors. Oral and maxillofacial surgery. 2 nd edn. St. Louis, MO: Saunders/Elsevier, 2009; p. 881-97.

16. Aggarwal VR, Lovell $K$, Peters $S$, Javidi $H$, Joughin A, Goldthorpe J. Psychosocial interventions for the management of chronic orofacial pain. Cochrane Database Syst Rev 2011;(11):CD008456. doi: 10.1002/14651858. CD008456.pub2.
17. Fricton J, Look JO, Wright E, et al. Systematic review and meta-analysis of randomized controlled trials evaluating intraoral orthopedic appliances for temporomandibular disorders. J Orofac Pain 2010;24(3):237-54.

18. Al-Ani MZ, Davies SJ, Gray RJ, Sloan P, Glenny AM. Stabilisation splint therapy for temporomandibular pain dysfunction syndrome. Cochrane Database Syst Rev 2004;(1):CD002778.

19. Pihut M, Ferendiuk E, Szewczyk M, Kasprzyk K, Wieckiewicz M. The efficiency of botulinum toxin type A for the treatment of masseter muscle pain in patients with temporomandibular joint dysfunction and tension-type headache. J Headache Pain 2016;17:29. doi: 10.1186/s10194016-0621-1.

20. Ahmed N, Poate T, Nacher-Garcia C, et al. Temporomandibular joint multidisciplinary team clinic. Br J Oral Maxillofac Surg 2014;52(9):827-30. doi: 10.1016/j. bjoms.2014.07.254.
21. Majakperuo HR, Watson M, Morrison R, Macfarlane TV. Pharmacological interventions for pain in patients with temporomandibular disorders. Cochrane Database Syst Rev 2010;(10):CD004715. doi: 10.1002/14651858. CD004715.pub2.

22. Al-Jamali JM, Voss PJ, Bayazeed BA, Spanou A Otten JE, Schmelzeisen R. Malignant tumors could be misinterpreted as temporomandibular joint disorders. Oral Surg Oral Med Oral Pathol Oral Radiol 2013;116(5):e362-67. doi: 10.1016/j. oooo.2012.01.039.

correspondence ajgp@racgp.org.au 